\title{
Percutaneous mitral valve repair with MitraClip for severe functional mitral regurgitation
}

\author{
Khung Keong $\underline{\text { Yeo }}^{1}$, mBвs, Zee Pin Ding ${ }^{1}$, MBвs, Yeow Leng $\underline{\text { Chua }}{ }^{2}$, MBвs, Soo Teik $\underline{\text { Lim }}^{1}$, MBBs, \\ Kenny Yoong Kong Sin${ }^{2}$, MBBs, Jack Wei Chieh $\underline{T a n}^{1}$, MBBs, Paul Toon Lim $\underline{C h i a m}^{1}$, MBBs, \\ Nian Chih Hwang $^{3}$, MBBs, Tian Hai Koh${ }^{1}$, MBBs
}

\begin{abstract}
A 67-year-old Chinese woman with comorbidities of chronic obstructive lung disease, hypertension and prior coronary artery bypass surgery presented with severe functional mitral regurgitation (MR) and severely depressed left ventricular function. She was in New York Heart Association (NYHA) Class II-III. Due to high surgical risk, she was referred for percutaneous treatment with the MitraClip valve repair system. This procedure is typically performed via the femoral venous system and involves a transseptal puncture. A clip is delivered to grasp the regurgitant mitral valve leaflets and reduce MR. This was performed uneventfully in our patient, with reduction of MR from 4+ to $1+$. She was discharged on post-procedure Day 2 and her NYHA class improved to Class I. This was the first successful MitraClip procedure performed in Asia and represents a valuable treatment option in patients with severe MR, especially those with functional MR or those at high surgical risk.
\end{abstract}

Keywords: heart failure, minimally invasive, mitral regurgitation

Singapore Med J 2013; 54(1): e9-e12

\section{INTRODUCTION}

MitraClip is a catheter-based device designed to perform edge-to-edge reconstruction of an insufficient mitral valve while the heart is beating, as an alternative to the conventional surgical approach. ${ }^{(1-3)}$ The procedure is performed in a cardiac catheterisation laboratory or operating room with echocardiographic and fluoroscopic guidance while the patient is under general anaesthesia. The device was CE marked in Europe in March 2008 and approved for use in Australia in March 2011. The Endovascular Valve Edge-to-Edge Repair Study (EVEREST II) randomised controlled trial that compared conventional surgery to the MitraClip showed that the device was less efficacious than surgery but was superior in safety. ${ }^{(4)}$ Both groups had similar improvements in left ventricular size, New York Heart Association (NYHA) functional class and quality of life. We describe here the first MitraClip procedure performed in Asia.

\section{CASE REPORT}

A 67-year-old Chinese woman with a history of congestive heart failure, coronary artery disease, chronic obstructive lung disease, hypertension and dyslipidaemia presented in 2008 with congestive heart failure and depressed left ventricular systolic function. Coronary angiography showed multivessel disease and she underwent coronary artery bypass surgery (CABG). She had an internal mammary artery graft to the left anterior descending artery and a radial artery graft to the right posterior descending artery. The patient recovered well from the CABG. However, she began to complain of increasing exertional dyspnoea two years later. Subsequent echocardiography showed the presence of severe mitral regurgitation (MR) and poor left ventricular ejection fraction (LVEF). The patient had been treated for heart failure and was on furosemide, bisoprolol, valsartan, aspirin, isosorbide mononitrate and atorvastatin. She was not offered cardiac resynchronisation therapy, as electrocardiography did not show evidence of intraventricular dyssynchrony. Despite appropriate medical therapy for her congestive heart failure, she remained in NYHA Class II-III.

Her echocardiography showed LVEF to be $25 \%$. There were regional wall motion abnormalities present, severe MR (Fig. 1) and moderate tricuspid regurgitation. The estimated pulmonary arterial systolic pressure was $43 \mathrm{mmHg}$. The left atrium was dilated, with a diameter of $57 \mathrm{~mm}$. The MR was likely due to left ventricular dilatation (functional MR) without evidence of intrinsic mitral leaflet disease. Transoesophageal echo (TEE) confirmed the presence of $4+$ functional MR with a central regurgitant jet. There was no evidence of mitral stenosis. Given her severe MR and change in clinical status, repeat coronary angiography was performed. The test revealed that only her internal mammary artery graft was patent, and she otherwise had extensive diffuse disease, with no further options for revascularisation. She was evaluated for possible mitral valve repair surgery. Pulmonary function tests showed her forced expiratory volume in 1 second (FEV1) to be $0.79 \mathrm{~L} / \mathrm{s}(56 \%)$, with a FEV1/FVC ratio of 0.73 . Her cardiothoracic surgeon felt that she was not suitable as an operative candidate due to her previous cardiac surgery, severe left ventricular dysfunction and lung disease. Given her symptoms, she was offered percutaneous repair of MR with the MitraClip valve repair system.

The procedure was performed by a multidisciplinary team comprising interventional cardiologists, echocardiologists, cardiac surgeons, cardiac anaesthesiologists, nurses and radiographers. The patient was placed under general anaesthesia and mechanically

${ }^{1}$ Department of Cardiology, ${ }^{2}$ Department of Cardiothoracic Surgery, ${ }^{3}$ Department of Cardiothoracic Anaesthesia, National Heart Centre Singapore, Singapore Correspondence: Dr Yeo Khung Keong, Consultant, Mistri Wing, National Heart Centre Singapore, 17 Third Hospital Avenue, Singapore 168752. yeo.khung.keong@nhcs.com.sg 
ventilated for the procedure. TEE was performed for baseline measurements and provided crucial imaging guidance throughout the procedure. Vascular access was obtained with an $8 \mathrm{~F}$ sidearm sheath in the right and left femoral veins, and a $6 \mathrm{~F}$ sheath in the left femoral artery. Right heart catheterisation was first performed to document right heart pressures. Transseptal puncture was then performed via the right femoral venous access under TEE guidance. The transseptal puncture is a critically important part of the MitraClip procedure, as it determines the approach of the clip into the mitral valve plane. Inappropriate location of the puncture can severely hamper manoeuvrability of the device.

Once the transseptal puncture was performed, the MitraClip guide was advanced into the left atrium. Next, we advanced the clip delivery system into the left atrium under TEE guidance and positioned the clip over the mitral valve, carefully avoiding the aorta and the left atrial wall. A variety of TEE views were obtained to guide the positioning of the clip across the regurgitant jet. The clip was then advanced with its arms in the open position into the left ventricle. Further adjustments were made with fluoroscopic and TEE guidance. Once the positioning was determined to be ideal, the mitral leaflets were grasped with real-time TEE guidance and the clip was closed (Fig. 2). The mitral valve was then interrogated for adequacy of leaflet capture, degree of remnant MR and evidence of mitral stenosis.

$M R$ in the patient was reduced from $4+$ to $1+$, the mean gradient across the dual orifice mitral valve was $2 \mathrm{mmHg}$ and leaflet capture was confirmed on multiple views. The clip was then released from the delivery catheter and final TEE images obtained (Fig. 3). Right and left heart catheterisation was repeated to document final haemodynamic parameters. The guide catheter was then removed and the right femoral venous puncture percutaneously repaired with Proglide devices (Abbott Vascular, Abbott Park, IL, USA). The total procedural time was 3 hours 17 minutes and the contrast volume (Omnipaque) was $70 \mathrm{~mL}$. The fluoroscopy time was 34.3 minutes with a dose area product of $18,063 \mathrm{mGym}^{2}$. The patient was transferred to the intensive care unit (ICU) where she was extubated within the subsequent hour. After an uneventful overnight stay in the ICU, she was transferred to the general ward and discharged on postoperative Day 2. The total length of stay was three nights, including the night prior to the admission. At the one-month follow-up, repeat echocardiography showed that MR remained at grade 1+. Six months post-procedure, repeat echocardiography showed grade $1-2+$ MR. The patient is now able to perform daily activities, including grocery shopping, independently. The patient is currently in NYHA Class I.

\section{DISCUSSION}

MitraClip is the first-in-class percutaneous therapy for mitral regurgitation (Fig. 4). It is a transcatheter technology based on the surgical techniques pioneered by Alfieri et al and emulates the surgical double-orifice approach to MR repair. ${ }^{(5-7)}$ The recently reported EVEREST II study showed favourable results.(4) This
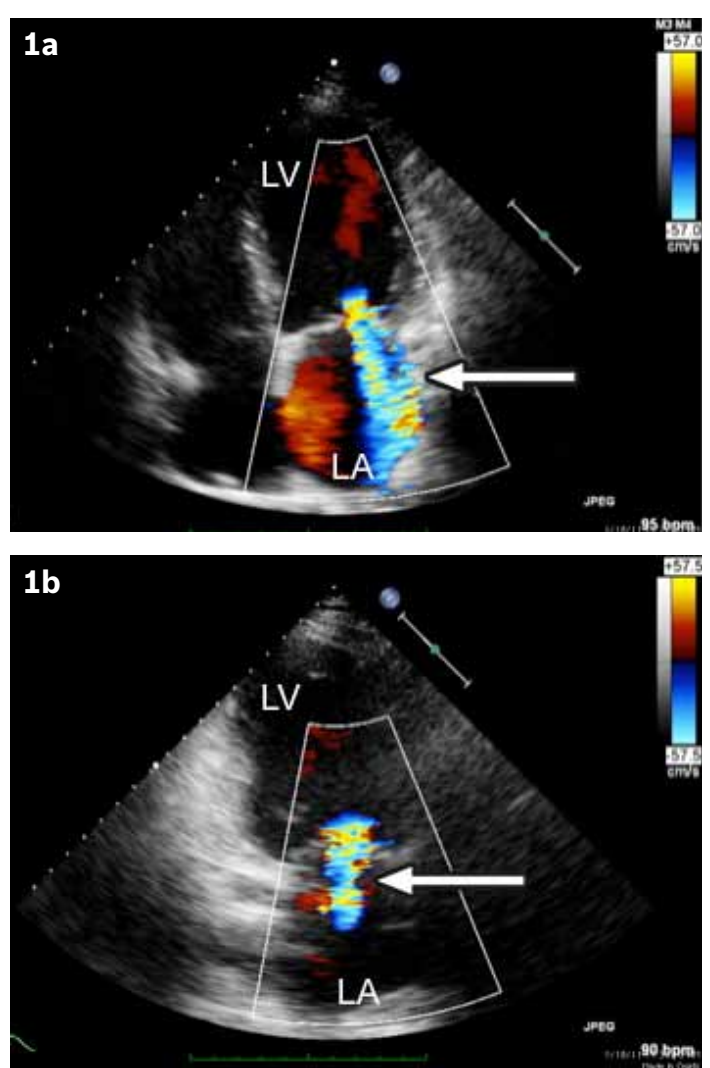

Fig. 1 Transthoracic colour Doppler echo images in the (a) apical fourchamber view and (b) apical two-chamber view demonstrate severe mitral regurgitation (white arrows) LV: left ventricle; LA: left atrium
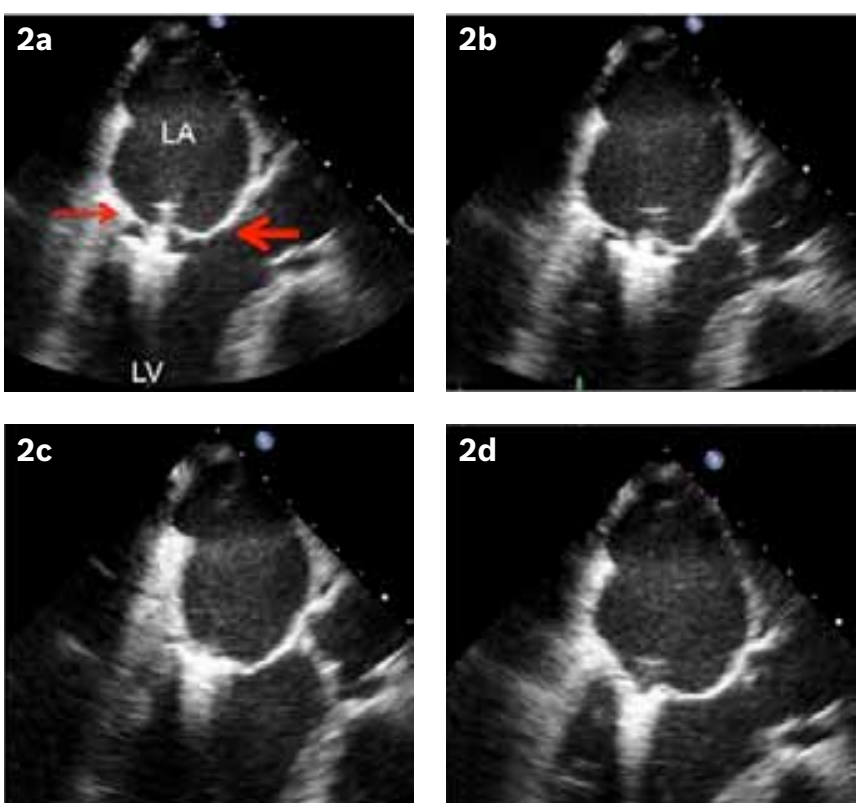

Fig. 2 Sequential transoesophageal echo images in the left ventricular outflow tract view on transoesophageal echo show the clip grasping the anterior (fat red arrow) and posterior (thin red arrow) leaflets of the mitral valve. LV: left ventricle; LA: left atrium

important randomised trial compared the use of MitraClip against surgery in patients with either severe degenerative (e.g. mitral valve prolapse) or functional MR. Enrolled patients had to meet the indications of the American College of Cardiology/American Heart Association guidelines for surgical treatment. The premise 

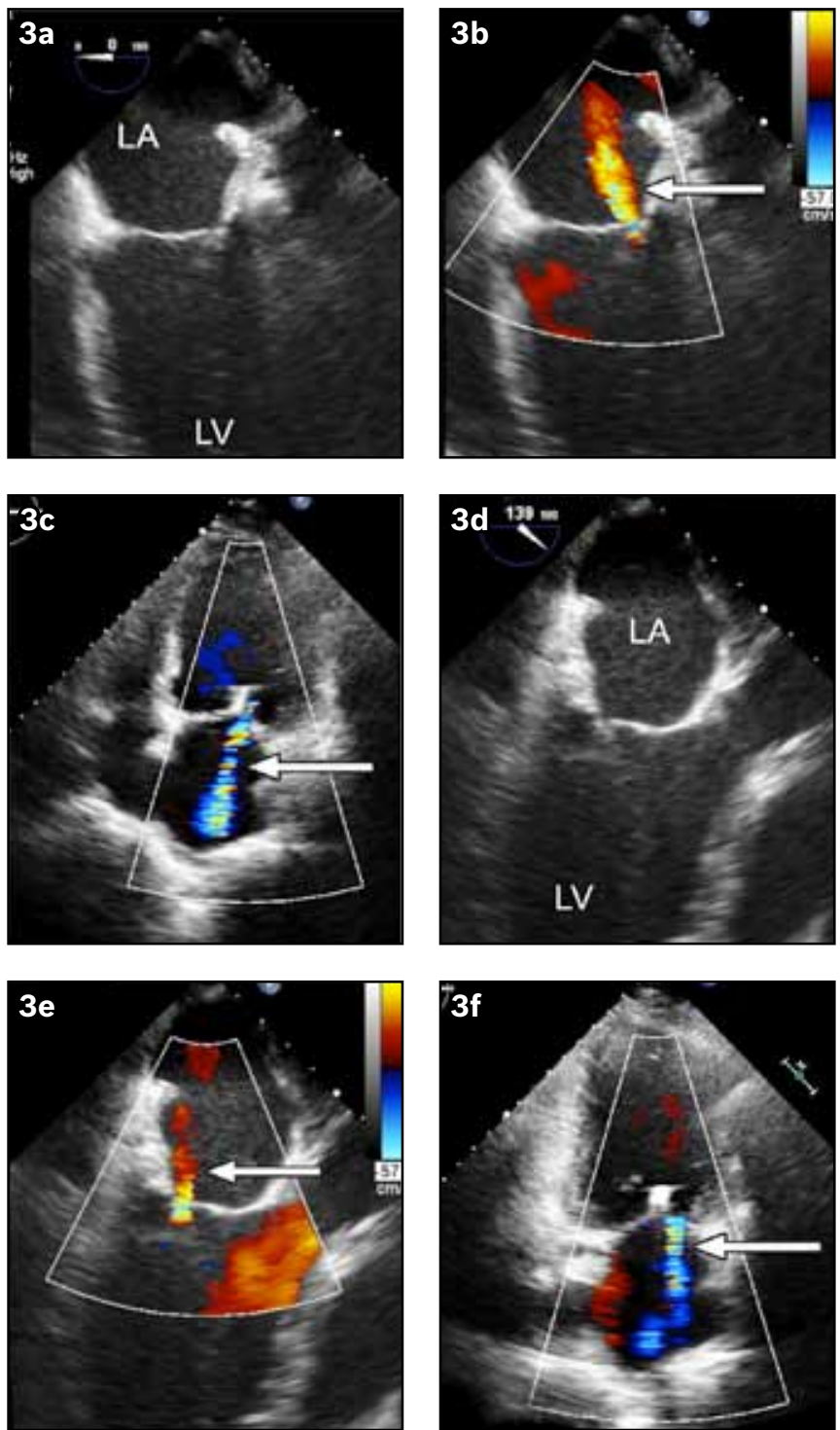

Fig. 3 Transoesophageal echo images at the conclusion of the procedure show the presence of mild residual mitral regurgitation. Images show the four-chamber view (a) without and (b) with colour Doppler. Images show the left ventricular outflow tract view (d) without and (e) with colour Doppler. (c \& f) Images show transthoracic echo images with colour Doppler at six months, demonstrating mild to moderate mitral regurgitation. The white arrows point to the residual mitral regurgitation jet. LV: left ventricle, LA: left atrium.

of the trial was that the MitraClip would be non-inferior to and safer than surgery. The primary composite end point for efficacy was freedom from (a) death; (b) surgery for mitral valve dysfunction; and (c) from grade 3+ or 4+ MR at 12 months. The primary safety end point was a composite of major adverse events within 30 days. The study found that although percutaneous repair was less effective at reducing MR than conventional surgery (although meeting its non-inferiority endpoint; $55 \%$ vs. $73 \%, p=0.007)$, the procedure was associated with superior safety $(15 \%$ vs. $48 \%, p<0.001)$ and similar improvements in clinical outcomes, such as NYHA functional class and quality of life. The device has gained early clinical acceptance in Europe as a treatment option for severe MR, but remains investigational in the USA. ${ }^{(8,9)}$ Our case report represents the first use of this therapy in Asia.

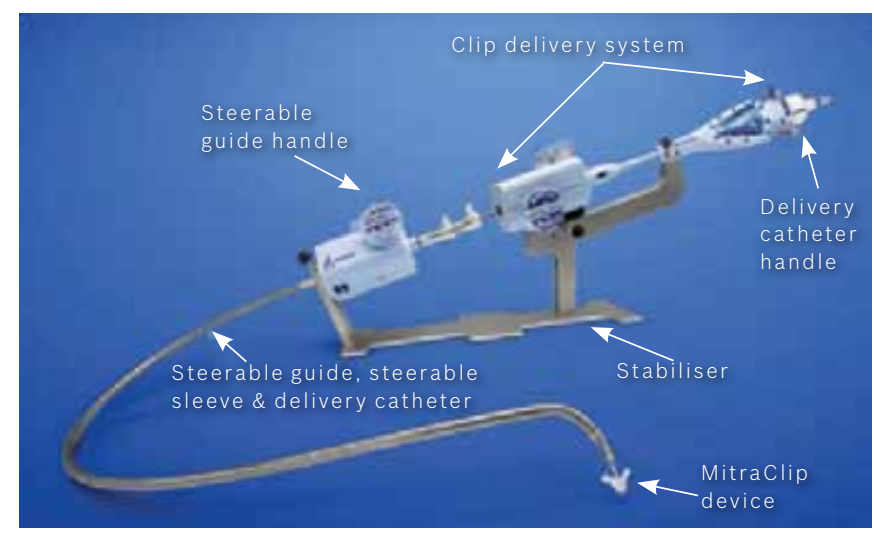

Fig. 4 Photograph shows the MitraClip repair system with its components labelled.

MitraClip comprises the clip itself, a clip delivery system, a guide and a stabiliser. The procedure is performed under general anaesthesia and primarily with TEE guidance, although some fluoroscopy is used as well. General anaesthesia is required because of the continuous use of TEE guidance and the need for the patient to remain completely still for the procedure..$^{(1,4)}$ MitraClip was originally designed for use in patients with degenerative MR. However, it has also been used in patients with functional MR, such as in our patient. In fact, worldwide use of the device is more common in patients with functional $M R .{ }^{(8,9)}$ The main reason for the greater use in patients with functional MR is because these patients often have significant risk, and open surgical repair/replacement has not been shown to improve mortality. Functional MR patients often have depressed left ventricular function. Many have underlying ischaemic heart disease and may have undergone prior coronary artery bypass surgery or percutaneous coronary intervention. These patients also tend to be older and have multiple comorbidities. These factors contribute to the procedural risk of any surgery. In contrast, the MitraClip procedure is remarkably safe. In the EVEREST II trial, which included both degenerative and functional MR patients, the MitraClip procedure was significantly safer than surgery. ${ }^{(4)}$ However, this difference in composite major adverse outcome was largely driven by ventilation time and the use of blood transfusions. Both are 'soft' adverse events. The rate of adverse events, such as mortality, stroke and myocardial infarction, was similar between the two arms.

Nonetheless, bleeding risks are substantially lower in MitraClip therapy, as it is performed percutaneously via the venous system. This is a considerable advantage for patients with significant surgical risk, as sternotomy and cardiopulmonary bypass are not advised for this cohort. It should be noted that in patients with functional MR, surgery has never been conclusively shown to provide mortality benefit when compared to medical therapy alone, although there appears to be functional and symptomatic improvements in some patients. ${ }^{(10-13)}$ So far, there has been no randomised trial for the comparison of MitraClip to medical therapy alone in functional MR patients. However, similar to surgical therapy, MitraClip has been shown to improve NHYA class status, quality of life and echocardiographic parameters 
(such as left ventricular chamber size) in patients with functional MR. ${ }^{(4)}$ However, while the results of the EVEREST II trial show that the therapy is generally safe, important complications may still occur. These complications include stroke, clip detachment of a single leaflet, mitral stenosis, bleeding, recurrence of MR and device malfunction. ${ }^{(4)}$ Although two-year results have been presented, longer term data remains unavailable.

It should be emphasised that the global experience with the MitraClip therapy remains limited compared to cardiac surgery. The majority of patients with degenerative MR should be treated with surgery if indications are met. Selecting the appropriate patient is an important part of the procedure. Firstly, clinical indications should be met. These include the development of symptoms of heart failure, a worsening LVEF and other evidence of left ventricular dysfunction, such as atrial fibrillation and pulmonary hypertension. Secondly, the risk of surgery should be assessed on a case-by-case basis. In patients with high surgical risk, procedures such as the MitraClip may be safer. Thirdly, the anatomic suitability of the patient for MitraClip needs to be carefully evaluated on echocardiography. In certain instances, the presence of excessively flail leaflets, mitral valve clefts, endocarditis or pre-existing concomitant mitral stenosis may make the device unsuitable for use. Due to its complexity and cost, and the presence of surgery as a well-established, efficacious therapy, potential candidates for MitraClip should be discussed in a multidisciplinary meeting to determine suitability.

MitraClip represents an exciting advancement in the arena of structural heart disease. It is the first percutaneous therapy available for severe MR and continues to be the only commercially available device in use in the Asia-Pacific region. It remains investigational in the USA, but has been in clinical use in Europe since 2008. For patients with severe MR requiring treatment and yet at high risk for surgical repair, MitraClip presents a safe, alternative therapeutic option.

\section{REFERENCES}

1. Feldman $T$, Kar $S$, Rinaldi $M$, et al. Percutaneous mitral repair with the MitraClip system: safety and midterm durability in the initial EVEREST (Endovascular Valve Edge-to-Edge REpair Study) cohort. J Am Coll Cardiol 2009; 54:686-94

2. Feldman T, Wasserman HS, Herrmann HC, et al. Percutaneous mitral valve repair using the edge-to-edge technique: six-month results of the EVEREST Phase I Clinical Trial. J Am Coll Cardiol 2005; 46:2134-40.

3. Franzen O, Baldus S, Rudolph V, et al. Acute outcomes of MitraClip therapy for mitral regurgitation in high-surgical-risk patients: emphasis on adverse valve morphology and severe left ventricular dysfunction. Eur Heart J 2010; 31:1373-81.

4. Feldman T, Foster E, Glower DD, et al. Percutaneous repair or surgery for mitral regurgitation. N Engl J Med 2011; 364:1395-406.

5. Alfieri O, Maisano F. An effective technique to correct anterior mitral leaflet prolapse. J Card Surg 1999; 14:468-70.

6. Fucci C, Sandrelli L, Pardini A, et al. Improved results with mitral valve repair using new surgical techniques. Eur J Cardiothorac Surg 1995; 9:621-6.

7. Maisano F, Torracca L, Oppizzi M, et al. The edge-to-edge technique: a simplified method to correct mitral insufficiency. Eur J Cardiothorac Surg 1998; $13: 240-5$

8. Franzen O, Baldus S, Rudolph V, et al. Acute outcomes of MitraClip therapy for mitral regurgitation in high-surgical-risk patients: emphasis on adverse valve morphology and severe left ventricular dysfunction. Eur Heart J 2010; 31:1373-81

9. Tamburino C, Ussia GP, Maisano F, et al. Percutaneous mitral valve repair with the MitraClip system: acute results from a real world setting. Eur Heart J 2010; 31:1382-9.

10. Bishay ES, McCarthy PM, Cosgrove DM, et al. Mitral valve surgery in patients with severe left ventricular dysfunction. Eur J Cardiothorac Surg 2000; 17:213-21.

11. Di Salvo TG, Acker MA, Dec GW, Byrne JG. Mitral valve surgery in advanced heart failure. J Am Coll Cardiol 2010; 55:271-82.

12. Kapoor JR. Role of mitral valve surgery for secondary mitral regurgitation in heart failure. J Am Coll Cardiol 2009; 53:74.

13. Wu AH, Aaronson KD, Bolling SF, et al. Impact of mitral valve annuloplasty on mortality risk in patients with mitral regurgitation and left ventricular systolic dysfunction. J Am Coll Cardiol 2005; 45:381-7. 